\title{
Neurological crisis in hereditary tyrosinaemia and complete reversal after liver transplantation
}

\author{
G Noble-Jamieson, N Jamieson, P Clayton, S Bailey, M Ryalls, N D Barnes
}

\begin{abstract}
A 19 month old Indian girl with tyrosinaemia developed a severe generalised neuropathy involving both phrenic nerves. Treatment with haemarginate failed to improve her condition. After liver transplantation the raised concentrations of the neurotoxin delta aminolaevulinic acid returned to normal and gradual but complete neurological recovery occurred over a period of 13 months.

(Arch Dis Child 1994; 70: 544-545)
\end{abstract}

Hereditary tyrosinaemia is a recessive disorder of amino acid metabolism caused by deficiency of fumarylacetoacetate hydrolase. The accumulation of tyrosine metabolites is thought to be the cause of the characteristic acute and/or chronic liver failure, renal tubular defects, and the high risk of eventual formation of hepatocellular carcinoma. Episodes of acute neuropathy, which clinically resemble porphyric crises, occur in up to $40 \% \%^{1}$ of affected children and may be so severe that mechanical ventilation for respiratory failure is necessary. Although most children who survive regain normal neurological function between crises, the mortality is high (up to $65 \%$ ).

\section{Case report}

A girl of 19 months with tyrosinaemia was referred with paralysis involving respiratory muscles and all four limbs. The diagnosis had been made at the age of 6 months and she was treated with an appropriate diet and made good developmental progress. However, she had become unwell one month before her admission with progressive weakness and breathlessness which lead to respiratory failure within two weeks. Electrophysiological studies demonstrated a severe demyelinating neuropathy affecting her arms more than her legs; brain stem studies were normal. She also had a renal tubular acidosis, was hypertensive and had greatly raised concentrations of $\alpha$ fetoprotein $(820 \mathrm{kU} / \mathrm{h}$, normal value less than 10$)$ but no evidence of hepatoma on magnetic resonance imaging. She was given a 14 day course of haemarginate (Normosang, Medica Pharmaceutical Company) which may benefit the neurological problem by inhibiting the formation of the neurotoxins delta aminolaevulinic acid (ALA) and porphobilinogen (PBG). ${ }^{2}$ As there was no clinical improvement it was decided that liver transplantation offered the only realistic hope of recovery. One previous report had recorded a successful outcome in a child with similar but less severe symptoms. ${ }^{2}$

She received an orthotopic graft of a whole donor organ on 18 January 1992 and had normal liver function from 10 days postoperatively. The explanted liver showed typical nodular cirrhosis with dysplastic changes but no tumour formation. The preoperatively increased ALA concentrations returned to normal two days after the operation and remained low (table). Neurological recovery was slow but progressive. With the help of a tracheostomy she was weaned from ventilation after seven weeks, began to talk and crawl after three months, and walked with a slight footdrop by five months. The tracheostomy was removed at seven months. Recovery of full power in all muscle groups had occurred by 13 months. Because of severe cyclosporin induced hypertrichosis she was changed to immunosuppression with FK506 (Fujisawa, Munich) and remains well nearly two years after transplantation with normal hepatic and renal function and normal nerve conduction studies.

\section{Discussion}

In hereditary tyrosinaemia liver transplantation offers the chance of 'cure' of the metabolic defect and also removes the risk of malignancy. Although common (affecting approximately $40 \%$ of children) recurrent, and carrying a high mortality, ${ }^{1}$ neurological complications in tyrosinaemia are not well recognised. The symptoms resemble those seen in porphyric crises with painful dysaesthesia, weakness, vomiting, ileus, and hypertension. Biochemically these crises are accompanied by a rise in serum ALA as a result of competitive inhibition of ALA dehydratase by succinylacetone, a metabolite of tyrosine degradation. Medical treatment with haemarginate suppresses the synthesis of ALA and PBG and

Serial measurements of urinary $A L A$ and PBG before and after transplantation

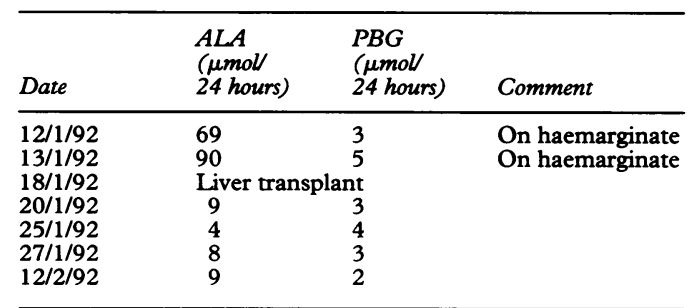

ALA reference range: $0-46 \mu \mathrm{mol} / 24$ hours.

PBG reference range: $0-9 \mu \mathrm{mol} / 24$ hours.

(These measurements were kindly done by Dr A C Deacon at the department of clinical chemistry, Northwick Park Hospital.) 
has been associated with improvement in porphyric crises. Rank et al described neurological improvement after treatment with haemarginate in a 21 month old boy with a neurological crisis. ${ }^{2}$ This child then received a liver transplant and did well. Our patient showed no improvement with haemarginate treatment and ALA concentrations only returned to normal after liver transplantation. With the severe demyelination demonstrated preoperatively, it was not surprising that neurological recovery was slow.

Recently a novel treatment with 2-(2-nitro-4trifluoro-methylbenzoyl)-1,3-cyclohexanedione has been shown to inhibit the tyrosine degradation pathway with marked reductions in succinylacetone, ALA, and PBG. $^{3}$ Experience with this compound is at present limited it is not yet clear what side effects the treatment may produce and whether neurological crises and the risk of hepatoma will be entirely eliminated.

1 Mitchell G, Larochelle J, Lambert M, et al. Neurologic crises in hereditary tyrosinemia. N Engl f Med 1990; 322: 432-7. 2 Rank JM, Pascual-Leone A, Payne W, et al. Clinical and laboratory observations. Hematin therapy for the neurologic oratory observations. Hematin therapy for the n

3 Lindstedt S, Holme E, Lock EA, Hjalmarson O, Strandvik B. Treatment of hereditary tyrosinemia type I by inhibition of 4-hydroxyphenylpyruvate dioxygenase. Lancet 1992; 340 813-7.

\section{High dose methylprednisolone for painful sickle cell crises}

The management of pain in sickling disorders was discussed in Archives in August 1993 (69: 256-9). Now doctors in Texas (Timothy C Griffin and colleagues, New England fournal of Medicine 1994; 330: 733-7) have described their experience with the use of high dose intravenous methylprednisolone in painful sickling crises. They studied 56 painful episodes in 36 patients aged from 2 to 19 years. In a placebo controlled, double blind study with random assignment methylprednisolone was used in 26 episodes and placebo in 30 . The dose of intravenous methylprednisolone was $15 \mathrm{mg} / \mathrm{kg}$ of body weight (maximum $1.0 \mathrm{~g}$ ) given over 30 minutes soon after admission to hospital and repeated 24 hours later.

Episodes in which placebo was given necessitated intravenous or oral analgesics for a mean of 71.3 hours; those in which methylprednisolone was used necessitated it for a mean of 41.3 hours $(p=0.03)$. When seven patients who developed the sickle cell chest syndrome were excluded from the analysis the difference was more significant $(62.5 v 31.0$ hours, $p=0.01)$. There is a suggestion that patients given methylprednisolone may be more likely to have further painful crises soon after discharge. Five patients were readmitted because of pain within eight days and of those four had been given methylprednisolone.

Clearly more studies are needed. High dose methylprednisolone may have a useful role but the possibility of rebound crises is worrying. 